\title{
The Study About Physical Activity for Subjects With Prevention of Benign Prostate Hyperplasia
}

\author{
Ho Won Lee, Shin Ah Kim', Ji Won $\mathrm{Nam}^{2}$, Mi Kyung Kim², Bo Youl Choi ${ }^{2}$, Hong Sang Moon \\ Department of Urology, Hanyang University College of Medicine, Seoul; \\ ${ }^{1}$ Institute for Health and Society, Hanyang University College of Medicine, Seoul; \\ ${ }^{2}$ Department of Preventive Medicine, Hanyang University College of Medicine, Seoul, Korea
}

Purpose: The number of benign prostatic hyperplasia (BPH) subjects has been increasing worldwide, and many studies have been conducted to determine the treatment that can delay drug therapy or surgery. Subsequently, most of these studies involved physical activity (PA) and associated factors. Therefore, we aimed to determine factors associated with BPH prevalence based on a review of past and present studies and to investigate the effect of a healthy lifestyle as a protective factor of BPH occurrence.

Methods: We selected 582 subjects aged $\geq 40$ years from an initial 779 subjects recruited from Gyeonggi, Yangpyeong, South Korea, during August 2009 to August 2011. Trained investigators surveyed International Prostate Symptom Score and demographic information, including PA and lifestyle questionnaire during face-to-face interviews; further, they performed digital rectal examination, rectal ultrasonography, and measured prostate-specific antigen levels. The statistical association between PA and $\mathrm{BPH}$ was analyzed by logistic regression analysis using multivariable regression models which use categorical variables by the Cochran-Mantel-Haenszel test and continuous variables by the general linear model.

Results: Seven statistically significant variables for PA were selected. Regular exercise, frequency of exercise, sedentary time, nonsedentary time, leisure time PA (metabolic equivalent, hr/wk) were not statistically associated with prostate volume but sedentary time (hr/day) was the only factor that showed a significant association in the multivariable model, including a linear effect relationship. Subjects with lower levels of sedentary time (4.5-7.0 hr/day) had a significantly lower risk of BPH (odds ratio [OR], 0.93; 95\% confidence interval [CI], 0.52-1.67) than those with a higher sedentary time (>7 hr/day) (OR, 1.72; 95\% CI, 0.96-3.09) (P for trend $=0.05$ ).

Conclusions: Our study showed that reducing sedentary time could have a protective effect and reduce the prevalence of BPH. Further prospective studies with a larger sample size are needed to assess the impact of reducing sedentary time on BPH risk.

Keywords: Prostatic hyperplasia; Leisure activities; Quality of life

\section{INTRODUCTION}

Although benign prostatic hyperplasia (BPH) is not a serious condition, it is a chronic disease associated with lower urinary tract symptoms (LUTS) such as narrow urine stream, residual urine sense, increased urinary frequency, and nocturia; these can cause considerable discomfort and affect the quality of life (QoL) in older men [1]. BPH has a high worldwide prevalence, with 612 million cases predicted to occur by 2018 ; the prevalence rates were $20 \%$ in Japan, $30 \%$ in the Netherlands, $40 \%$ in Korea, and
$50 \%$ in United States (US) [2]. BPH prevalence increases with age, and over half of the male population will have $\mathrm{BPH}$ by the time they reach middle age [3]. BPH-estimated socioeconomic burden is expected to increase globally [3-5]. In the US, the direct medical costs associated with $\mathrm{BPH}$ are currently over 1.1 billion US Dollars annually and are expected to rise [6].

Age-related changes in the anatomically enlarged prostate are thought to be responsible for the LUTS. The sympathetic nervous system release causes an increase in prostate smooth muscle tone, subsequently narrowing the urethra [7]. The gradual worsening
Corresponding author: Shin Ah Kim

Department of Urology, Hanyang University College of Medicine,

222 Wangsimni-ro, Seongdong-gu, Seoul 133-791, Korea

TEL: +82-2-2220-0660 / Fax: +82-2-2293-0660 / E-mail: gdindus@hanmail.net

Submitted: July 13, 2014 / Accepted after revision: August 31, 2014
This is an Open Access article distributed under the terms of the Creative Commons Attribution Non-Commercial License (http://creativecommons.org/licenses/by-nc/3.0/) which permits unrestricted non-commercial use, distribution, and reproduction in any medium, provided the original work is properly cited. 
of LUTS results in further reduction of the QoL [8]. Pharmacotherapy with 5 -alpha reductase inhibitors or alpha-1 blockers is frequently used to alleviate LUTS, but their continuous administration and the need for increasingly higher doses presents disadvantages, and symptom resolution is not always satisfactory [9].

Age, genetic, and hormonal factors have been traditionally considered as the main risk factors for $\mathrm{BPH}$ and its associated pathophysiology, but lifestyle elements such as PA, leisure, exercise, smoking, and drinking are now considered to play an important role in the development of BPH [10-13]. The importance of PA in the prevention of chronic diseases, including metabolic syndrome, has been suggested from several epidemiologic studies in which PA was shown to have a protective effect. Furthermore, a lifestyle that includes moderate levels of leisure-time such as that involving PA was associated with a lower risk of BPH or less severe LUTS [12,14-16]. From the long-term point of view, pharmacotherapy is likely to have a socioeconomic impact in the elderly population because of their lower economic ability and the need for continuous medication [9]. Inexpensive prevention measures, such as increasing PA levels, appear an attractive risk reduction strategy that could prove to be more cost-effective than pharmacotherapy or surgery for the treatment of $\mathrm{BPH}$ in older men.

In this study, we aimed to investigate the effects of PA on the risk of BPH in men aged $\geq 40$ years living in Gyeonggi, Yangpyeong, South Korea.

\section{MATERIALS AND METHODS}

This study was approved by the Institutional Review Board (IRB) of Hanyang University College of Medicine. IRB numbers were HYUH IRB 2010-R-38 and 2011-07-005.

Subjects aged $\geq 40$ were identified from the database of the Yangpyeong cohort study in the Gyeonggi Yangpyeong County that had recruited 779 subjects. All subjects were cohort participants, and a cross-sectional study was performed for the analysis of voiding difficulty because voiding difficulty examination was done for once.

A cross-sectional survey was performed every year in from August 2009 to August 2011. A questionnaire, modified from a PA questionnaire used for the Stanford Five-city project [17] was administered by trained interviewers during face-to-face interviews to collect demographic information as well as smoking, drinking, diet, and PA habits. LUTS were assessed using the International Prostate Symptom Score (IPSS). Men with urinary symptoms underwent a digital rectal examination (DRE) by a trained urologist, prostate-specific antigen (PSA) screening, and rectal ultrasonography for prostate size to distinguish prostate cancer from acute prostatitis. Exclusion criteria were history of prostate cancer $(n=2)$, unperformed rectal ultrasonography ( $n$ $=83$ ), incomplete of IPSS ( $n=6)$, missing value of other BPHrelated factors $(n=14)$, incomplete PA questionnaires $(n=21)$, missing information of other epidemiologic variables $(n=3)$, palpable nodules during DRE ( $\mathrm{n}=23$ ), PSA level $\geq 4.0 \mathrm{ng} / \mathrm{mL}$ $(\mathrm{n}=47)$. Among these, 2 subjects had PSA levels $\geq 4.0 \mathrm{ng} / \mathrm{mL}$ as well as nodules. As a result, a total of 582 subjects were selected as participants (Fig. 1).

BPH was defined as $\geq 25 \mathrm{~mL}$ of prostate volume and an IPSS score of $\geq 8$. Each PA level was estimated from the average number of hours spent per day per year, and applied to the seven variables of PA, based on the questionnaire, which had been used successfully in other studies $[12,17,18]$.

Regular exercise was judged by the absence or presence of sufficient amount of body sweat with exercise, exercise frequency (time/wk) was regarded as the exercise frequency per week, and exercise time (minute/once) was the average time used during one exercise. Sedentary time (hr/day) was the mean time the participants spent sitting during the day, including the sum of time participants spent sitting at home, during transportation, watch-

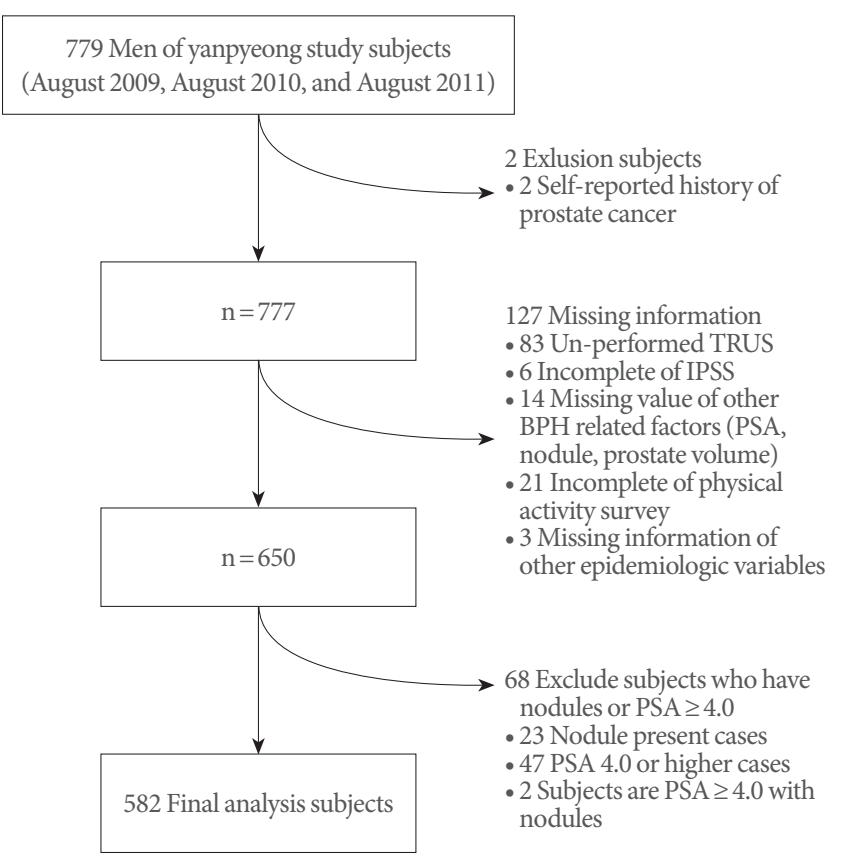

Fig. 1. Exclusion criteria. TRUS, transrectal ultrasound; IPSS, International Prostate Symptom Score; PSA, prostate-specific antigen. 
ing TV, mealtimes, sitting time outside of work hours. Nonsedentary time (occupational or leisure time, hr/day) was included in the time of intense activity as daily average working time or hours of exercise such as light field work, slow cycling, or walking. Activities such as planting; wiping windows; and energy spent while running, hiking, or climbing stairs as moderate PA. Leisure time was defined as the average daily exercise time and percentage of time spent during moderate exercise (walking, bowling, playing table tennis, doing yard work, or dancing) and vigorous exercise such as swimming and climbing. Leisure time physical activity (LTPA) was defined as average metabolic equivalent (MET) values of exercise time in two weeks, after which the exercise content was calculated as the energy consumed during activity for each movement. In addition, we calculated the total energy consumed as the average exercised time per day. LTPA was divided into two groups based on 22.5 METs, which is the minimum level of energy converted and consumed in a week, as recommended by the World Health Organization standard [18]. Categorical variables were analyzed by the Cochran-MantelHaenszel test and continuous variables by general linear models. To assess confounding, multivariable linear and logistic regression models were constructed and age-adjusted models were created to assess correlations between each PA variable and $\mathrm{BPH}$. Variables significantly associated with each PA variable were selected for inclusion in the multivariable models. In all analyses, the age-adjusted means and odds ratios (ORs), 95\% confidence intervals (95\% CIs), standard error, P-value, and P-for trend were presented.

\section{RESULTS}

Among the 582 subjects selected, 114 were diagnosed with $\mathrm{BPH}$. The mean age was $62.94 \pm 0.43$ years in the non-BPH group and $68.60 \pm 0.80$ years in the BPH group. In this study, age was statistically associated with $\mathrm{BPH}(\mathrm{P}<0.001)$. The associations between PA and other risk factors are shown in Tables 1, 2. Variables significantly associated with PA were selected based on the P-values obtained for inclusion in the multivariable models.

Sedentary time (hr/day), nonsedentary time (hr/day), and leisure time (hr/day) were subdivided and analyzed in 3 groups (low, medium, and high) based on the 33th percentile of the subjects' standard distribution. There was a significant trend between $\mathrm{BPH}$ and sedentary time, nonsedentary time, and leisure time, and $\mathrm{BPH}$ was higher among those with the highest sedentary time. Analysis of LTPA in all subjects showed that those with
LTPA $>22.5$, had significantly larger prostate volumes $(\mathrm{P}=0.024$, P for trend $<0.001$ ) (Table 2).

Multivariable logistic regression analyses were built to analyze correlations between PA variables and $\mathrm{BPH}$, controlling for confounding. The group with the lowest level of PA was selected as reference group to calculate ORs for all variables.

Regular exercise was a significantly associated risk factor for BPH (P-value 0.009; OR, 1.12; 95\% CI, 0.66-1.88). Those who exercised $\geq 5$ per week might be considered at a higher risk (OR, 1.26; 95\% CI, 0.68-2.33), but a significant linear relationship was not observed ( $\mathrm{P}$ for trend $=0.52$ ). Exercising for at least 60 minutes may be regarded as a risk factor (OR, 1.05; 95\% CI, 0.60-1.97), but it was not associated with an overall trend ( $\mathrm{P}$ for trend $=0.78$ ). Subjects with lower levels of sedentary time (4.5-7.0 hr/day) had a significantly lower risk of $\mathrm{BPH}(\mathrm{OR}, 0.93$; 95\% CI, 0.52-1.67) than those with a higher sedentary time ( $>7 \mathrm{hr} /$ day) $(\mathrm{OR}, 1.72$; 95\% CI, 0.96-3.09) (P for trend =0.05). Moreover, subjects with less sedentary time (1.79-3.6 hr/day) had a lower risk (OR, 0.61; 95\% CI, 0.34-1.07) of BPH than the group with $>3.6 \mathrm{hr} /$ day sedentary times (OR, 0.69; 95\% CI, 0.38-1.26), but this difference was not statistically significant. Those with lower leisure time (0.06-0.54 $\mathrm{hr} /$ day) were at an increased risk of BPH (OR, 0.95; 95\% CI, 0.501.82) compared to the higher leisure time group (OR, 0.89; $95 \% \mathrm{CI}$, 0.51-1.54), but this association was not statistically significant. In terms of LTPA, no statistically significant differences were observed when comparing the group with $\geq 22.5$ METs with the group with <22.5 (OR, 0.94; 95\% CI, 0.53-1.67) (Fig. 2).

\section{DISCUSSION}

In the past years, numerous studies have been carried out to identify risk factors associated with the etiology and pathogenesis of $\mathrm{BPH}[10,12,19,20]$. Rohrmann et al. [12] aimed to identify the relationship between smoking and $\mathrm{BPH}$, but there was no statistical association. Similarly, the link between alcohol consumption and chronic diseases such as diabetes mellitus, hypertension, body mass index, obesity, and dyslipidemia did not reveal an association [21-23].

The results of several studies designed to define the relationship between PA and BPH could be classified into three types: linear, inversed, and independent. In a review of 11 studies by Parsons and Kashefi [11], after limiting searches to keywords such as BPH, LUTS, and PA, 8 studies reported an inversely proportional relationship between $\mathrm{PA}$ and $\mathrm{BPH}$, whereas 2 studies reported no relationship and one reported an equal relationship. 


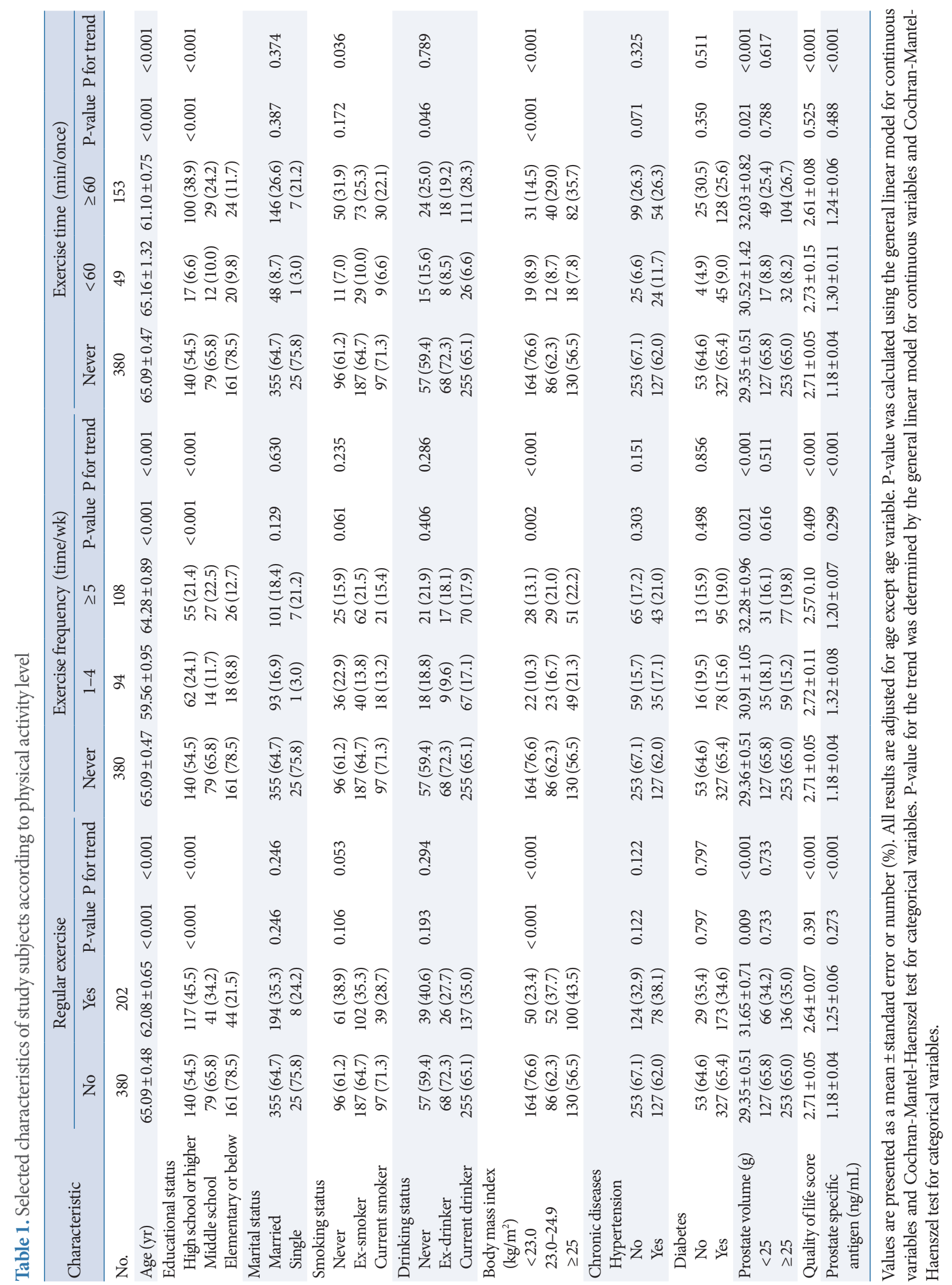




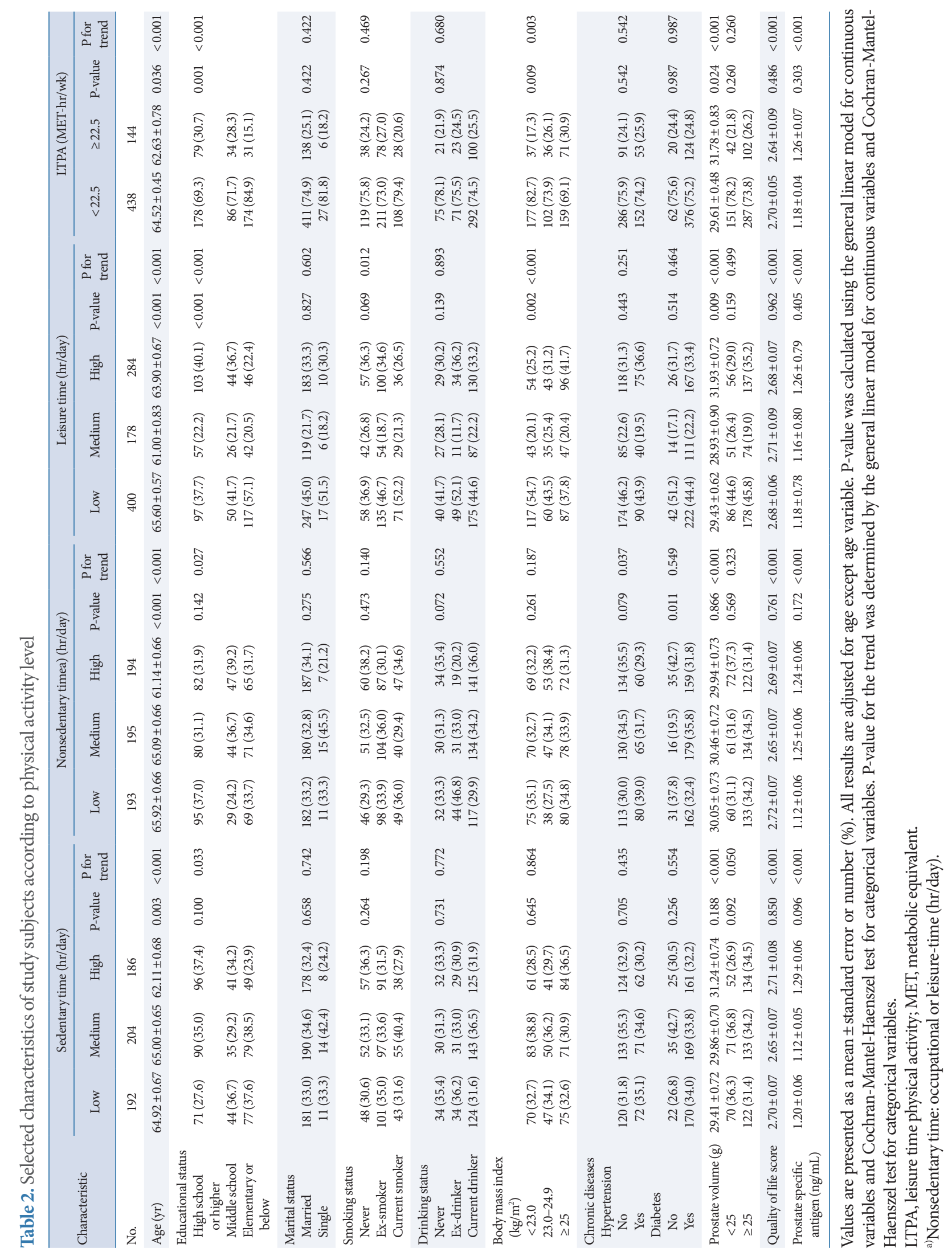




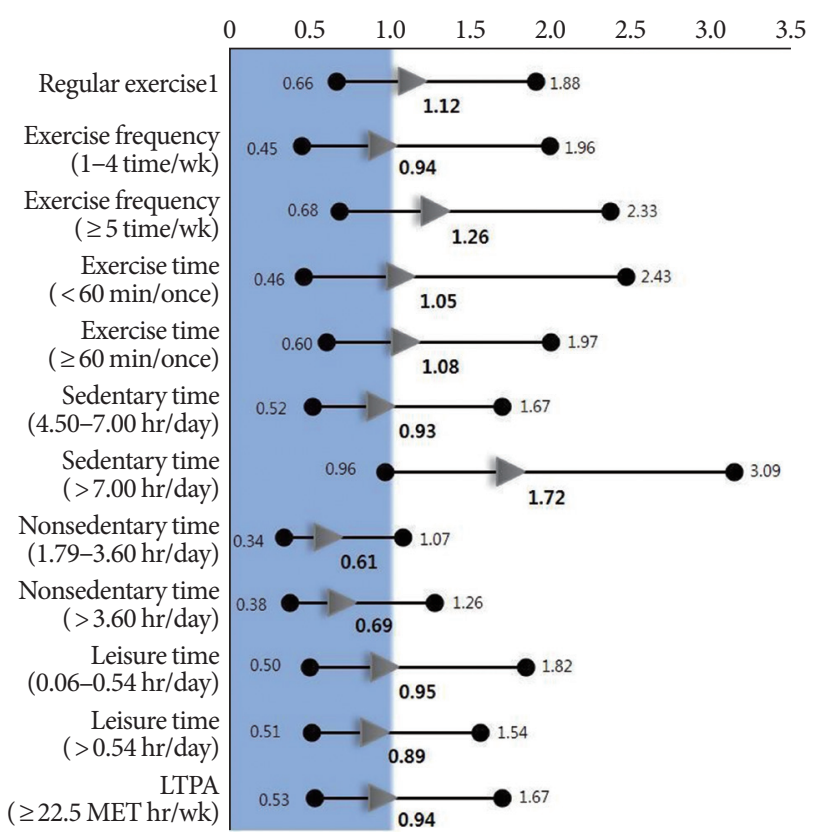

Fig. 2. Relation between physical activity level and benign prostatic hyperplasia. PSA, prostate-specific antigen; LTPA, leisure time physical activity; MET, metabolic equivalent.

All of those 8 studies investigated effects for $\mathrm{BPH}$ according to PA intensity and frequency and reported the greater effect with more intense and frequent PA [11]. However, the relationship between higher-strength and higher-frequency PA is not linearly proportional. In connection with this subject, excessive PA (daily motion or long-time physical activity ( $\geq 90 \mathrm{~min} /$ day) does not guarantee an improvement in individual health, which rather tends to decrease slightly [24]. The study which was reported relationships between PA and symptomatic BPH who underwent surgery in 1980. In this study, all subjects participated in athletic activities that were stratified on exercise intensity over thirteen years. Lowintensity activity as defined the "walking group" showed a statistically significant effect $(\mathrm{P}<0.001)$ in the improvement of $\mathrm{BPH}$ symptoms. The same study showed patients who have more $\mathrm{BPH}$ symptoms watched TV more than patients who have fewer symptoms [18]. However, our results are different from those of existing studies. BPH showed a statistically significant increase associated with regular exercise, but the frequency of exercise (time/wk) and exercise time (min/once), which corresponded to detailed classifications of PA, were not significantly associated with BPH risk. Similarly, leisure time (hr/day) was not a statistically significant risk factor for $\mathrm{BPH}$. Therefore, regular exercise was insufficient to generalize PA as a protective factor against the risk of BPH. A static time (sedentary time, hr/day), defined as the time sitting while doing domestic work and sitting in the office, and the time taken to exercise was higher and significantly associated with $\mathrm{BPH}$ risk. Comparatively, the higher the daily average PA levels per day, the higher the risk of $\mathrm{BPH}$, but this result did not reach statistical significance, because these activity time as "nonsedentary time" could include possibilities which affected on BPH. Additionally, LTPA was not a statistically meaningful risk factor for $\mathrm{BPH}$.

Platz et al. [18] have suggested potential physiological mechanisms that may be associated with reduced sedentary time and lower BPH risk. Reducing inactivity and sustaining PA can lower the systemic sympathetic nervous system activity, subsequently reducing prostatic smooth muscle tone and improving LUTS.

The hypothesized protective effect of high-intensity and longer duration exercise on the risk of $\mathrm{BPH}$ has been the subject of numerous studies since 1998 [8,20,25]. In our study, there was no statistically meaningful relationship between all possible variables of PA and BPH risk, but the life-time proportion of sitting time appeared to significantly increase BPH risk. This result has potential implications for the prevention of $\mathrm{BPH}$ in increasingly aging societies in which rates of $\mathrm{BPH}$ are gradually increasing.

Vignozzi et al. [25] demonstrated that the pathological mechanism of BPH is similar to that of metabolic syndrome to such an extent as to be considered "the new metabolic syndrome of 2014". The fact that lifestyle modification has a protective effect in the metabolic syndrome has already been proven [25]. In this study, reduced sedentary time, which had a statistically significant protective effect on $\mathrm{BPH}$, can be regarded as a lifestyle factor amenable to modification. Other studies have reported that high intensity exercise, long time exercise could help in reducing BPH risk, but in the present study, reduced sedentary time, which was statistically meaningful in BPH prevention could be easily implemented by older men. Although pharmacotherapy and surgery can improve LUTS, reducing sedentary time as a life style modification, is easy to practice and may help to reduce the risk of $\mathrm{BPH}$.

Our study has several limitations: because of our cross-sectional study design, the relationship between variables may be causal. Statistical power might be weak because physicians identified BPH from subjects who were already diagnosed in one population as part of the cohort. In other words, the relationship between reduced sedentary time and BPH could have been affected. This possibility that subjects who were already diagnosed with $\mathrm{BPH}$ had already maintained their lifestyle factors like re- 
ducing sedentary time might weaken statistical power. A possibility which were mixed newly diagnosed also did.

In conclusion, BPH prevalence of 60 years old men were reported more than half. And BPH treatment has needed economic efficiency and safety in elderly men. Furthermore, many studies have reported that various factors such as age, PA, genetics, and life style contribute to $\mathrm{BPH}$ and its associated symptoms. Among the many factors associated with $\mathrm{BPH}$, reducing sedentary time is expected to have a protective effect for $\mathrm{BPH}$. However, more research is needed in the future.

\section{CONFLICT OF INTEREST}

No potential conflict of interest relevant to this article was reported.

\section{REFERENCES}

1. Parsons JK. Modifiable risk factors for benign prostatic hyperplasia and lower urinary tract symptoms: new approaches to old problems. J Urol 2007;178:395-401.

2. Park HK, Park H, Cho SY, Bae J, Jeong SJ, Hong SK, et al. The prevalence of benign prostatic hyperplasia in elderly men in Korea: a community-based study. Korean J Urol 2009;50:843-7.

3. McVary KT. BPH: epidemiology and comorbidities. Am J Manag Care 2006;12(5 Suppl):S122-8.

4. Irwin DE, Kopp ZS, Agatep B, Milsom I, Abrams P. Worldwide prevalence estimates of lower urinary tract symptoms, overactive bladder, urinary incontinence and bladder outlet obstruction. BJU Int 2011;108:1132-8.

5. Groves HK, Chang D, Palazzi K, Cohen S, Parsons JK. The incidence of acute urinary retention secondary to BPH is increasing among California men. Prostate Cancer Prostatic Dis 2013;16:260-5.

6. Hollingsworth JM, Wilt TJ. Lower urinary tract symptoms in men. BMJ 2014;349:g4474.

7. Lepor H. Pathophysiology, epidemiology, and natural history of benign prostatic hyperplasia. Rev Urol 2004;6 Suppl 9:S3-10.

8. Caine M. The present role of alpha-adrenergic blockers in the treatment of benign prostatic hypertrophy. J Urol 1986;136:1-4.

9. Parsons JK, Im R. Alcohol consumption is associated with a decreased risk of benign prostatic hyperplasia. J Urol 2009;182:1463-8.

10. Cui Y, Zong H, Yan H, Li N, Zhang Y. Degarelix versus goserelin plus bicalutamide therapy for lower urinary tract symptom relief, prostate volume reduction and quality of life improvement in men with prostate cancer: a systematic review and meta-analysis. Urol
Int 2014;93:152-9.

11. Parsons JK, Kashefi C. Physical activity, benign prostatic hyperplasia, and lower urinary tract symptoms. Eur Urol 2008;53:1228-35.

12. Rohrmann S, Crespo CJ, Weber JR, Smit E, Giovannucci E, Platz EA. Association of cigarette smoking, alcohol consumption and physical activity with lower urinary tract symptoms in older American men: findings from the third National Health And Nutrition Examination Survey. BJU Int 2005;96:77-82.

13. Seim A, Hoyo C, Ostbye T, Vatten L. The prevalence and correlates of urinary tract symptoms in Norwegian men: the HUNT study. BJU Int 2005;96:88-92.

14. Schoenfeld MJ, Shortridge EF, Gelwicks SC, Cui Z, Wong DG. Treatment patterns in alpha-blocker therapy for benign prostatic hyperplasia. Am J Mens Health 2014;8:267-72.

15. Meigs JB, Mohr B, Barry MJ, Collins MM, McKinlay JB. Risk factors for clinical benign prostatic hyperplasia in a community-based population of healthy aging men. J Clin Epidemiol 2001;54:935-44.

16. Giovannucci E, Rimm EB, Chute CG, Kawachi I, Colditz GA, Stampfer MJ, et al. Obesity and benign prostatic hyperplasia. Am J Epidemiol 1994;140:989-1002.

17. Sallis JF, Haskell WL, Wood PD, Fortmann SP, Rogers T, Blair SN, et al. Physical activity assessment methodology in the Five-City Project. Am J Epidemiol 1985;121:91-106.

18. Platz EA, Kawachi I, Rimm EB, Colditz GA, Stampfer MJ, Willett WC, et al. Physical activity and benign prostatic hyperplasia. Arch Intern Med 1998;158:2349-56.

19. Speakman M, Kirby R, Doyle S, Ioannou C. Burden of male lower urinary tract symptoms (LUTS) suggestive of benign prostatic hyperplasia (BPH) - focus on the UK. BJU Int 2014 Mar 24 [Epub]. http://dx.doi.org/10.1111/bju.12745.

20. Hootman JM. 2008 Physical Activity Guidelines for Americans: an opportunity for athletic trainers. J Athl Train 2009;44:5-6.

21. Gass R. Benign prostatic hyperplasia: the opposite effects of alcohol and coffee intake. BJU Int 2002;90:649-54.

22. Gacci M, Corona G, Vignozzi L, Salvi M, Serni S, De Nunzio C, et al. Metabolic syndrome and benign prostatic enlargement: a systematic review and meta-analysis. BJU Int 2014 Mar 6 [Epub]. http://dx.doi. org/10.1111/bju.12728.

23. Abdollah F, Briganti A, Suardi N, Castiglione F, Gallina A, Capitanio $U$, et al. Metabolic syndrome and benign prostatic hyperplasia: evidence of a potential relationship, hypothesized etiology, and prevention. Korean J Urol 2011;52:507-16.

24. Brown DW, Brown DR, Heath GW, Balluz L, Giles WH, Ford ES, et al. Associations between physical activity dose and health-related quality of life. Med Sci Sports Exerc 2004;36:890-6. 


\section{Lee, et al. • Physical Activity for Subjects With Prevention of BPH}

25. Vignozzi L, Rastrelli G, Corona G, Gacci M, Forti G, Maggi M. Benign prostatic hyperplasia: a new metabolic disease? J Endocrinol
Invest 2014 Jan 24 [Epub]. http://dx.doi.org/10.1007/s40618-0140051-3. 